\title{
AN IMPROVEMENT ON TRAFFIC ASSIGNMENT METHOD
}

\author{
Shogo KAWAKAMI and ZhengKai LIU \\ Department. of Civil Engineering, School of Engineering, Nagoya University \\ Furocho Chikusaku, Nagoya 464-01 JAPAN
}

\begin{abstract}
Though the OD traffic flow is the traffic flow from one zone to another zone, the current traffic assignment methods assume that the OD traffic flow is generated from one node and destined to one node known as centroid. The shape and the constitution of zone are not taken into consideration at all in the assignment methods. In this paper, the errors caused by this assumption will be analyzed. Two traffic assignment methods will be developed to improve the current methods. In the improved methods, OD traffic flow can be generated from many or all nodes in any zone, but the needed data, computation and memory space are the same as that of general traffic assignment methods. The fitness of the improved methods will be certified by the traffic assignment of a assumed network and the road network of Nagoya city.
\end{abstract}

\section{Introduction}

The transportation planning process for urban area is typically based on a partition of area into traffic zone, and any zone generally contains many nodes and links. In the real traffic network, the traffic may be generated from any node in the zone, however, the current traffic assignment methods assume that the OD traffic flow in a zone is generated from one node known as centroid. The drawbacks of this assumption are manifold. First, it will cause that more traffic flow is assigned to the links which are near to the centroid and less traffic flow is assigned to the links which are far from the centroid. Second, the number of routes to which OD traffic flow may be loaded in an assignment procedure will be less than the number of routes that may be chosen by drivers in the actual traffic network because the feasible routes between two centroids are generally less than that between two zones. Third, the location of tha centroid in each zone has to be determined carefully before traffic 
assignment because different location of centroid will produce different assignment results. If the zone is divided to be very small, the errors produced by this assumption may be decreased, but the needed data, calculation and computer memory will increase dramatically.

In another aspect, in order to represent the phenomena that traffic can be generated anywhere in urban road network, researchers also developed some traffic assignment methods by continuum approximation of network. Although not few papers on this research were presented recently, there are still many theoretical and computational problems that remain to be solved for continuum models. It is difficult to apply continuum models to solve the practical traffic assignment problem in near future.

In this paper, two traffic assignment methods will be developed to improve the traditional traffic assignment methods on the assumption of traffic generation. In the improved methods, OD traffic flow can be generated from many or all nodes in any zone. The needed data, computation and memory space of the improved methods are almost the same as that of general traffic assignment method. In order to develop the method, two knds of shortestpath and the corresponding shortest-path algorithm were also introduced. The fitness of the improved methods will be certified by an assumed network and an actual network. From the calculation results, it can be seen that the improved methods are much better than the current traffic assignment method.

\section{Three Kinds of Shortest-path in Traffic Network}

It is known that any traffic assignment procedures assign OD traffic flow based on the shortest-path, that is, always assign a proportional of OD traffic flow to the shortest-path between origin and destination in one iteration. The shortest-path used in traditional assignment method is the shortest-path from the centroid of origin zone to the centroid of destination zone. We call this kind of shortest-path as centroid-to-centroid shortest-path. The assignment methods based on this kind of shortest-path actually transferred the traffic flow between two zones into the traffic flow between two centroids, however, the shape and the size of the zones was not taken into consideration at all. This can not be regarded to be reasonable. In order to take the size and shape of zone into consideration to some extent in assignment procedures, we define two new kinds of shortest-path based on zone, and then develop corresponding traffic assignment methods based on new shortest-path. We call them as node-to-zone shortest-path and zone-to-zone shortest-path.

First, we define the following symbols :

$S N_{i}$ : the set of nodes in zone $I$.

$S T_{k j}$ : the shortest travel cost from node $K$ to zone $J$.

$L_{i j}$ : the shortest travel cost from zone $I$ to zone $J$. 


\section{AN IMPROVEMENT ON TRAFFIC ASSIGNMENT METHOD}

$N T_{k l}$ : the shortest travel cost from node $K$ to node $L$

The definitions of node-to-zone shortest-path and zone-to-zone shortest-path are given as follows.

The shortest-path from a node to a zone: The shortest-path from node $K$ to zone $J$ is the shortest one among the shortest-path from node $K$ to each node in zone $J$, that is :

$$
S T_{k j}=\min _{1 \in S N_{j}} N T_{k l}
$$

Here, we call it node-to-zone shortest-path.

If we assume that the number of nodes in zone $J$ is $n$, then the number of shortest-paths from node $K$ to each of the node $L\left(L \in S N_{j}\right)$ is also $n$. The node-to-zone shortest-path from node $K$ to zone $J$ is the shortest one among the $n$ shortest-paths.

The shortest-path from a zone to another zone: The shortest-path from zone $I$ to zone $J$ is the shortest-path from each node in zone $I$ to each node in zone $J$, that is

$$
L_{i j}=\min _{k \in S N_{i}, 1 \in S N_{j}} N T_{k l}=\min _{k \in S N_{i}} S T_{k j}
$$

Here, we call it zone-to-sone shortest-path.

If we assume that the number of nodes in zone $J$ is $n$ and the number of nodes in zone $I$ is $m$, then the number of shortest-paths from each node $K\left(K \in S N_{i}\right)$ to each node $L\left(L \in S N_{j}\right)$ is $n \times m$. The zone-to-zone shortest-path from zone $I$ to zone $J$ is the shortest one among the $n \times m$ shortest-paths.

Generally, there exists,

$$
L_{i j} \leqq S T_{k j} \leqq N T_{k l} \quad \forall k \in S N_{i} \quad l \in S N_{j}
$$

Since the calculations of these two kinds of shortest-path are based on all the nodes in the zone, it should be more reasonable to assign the OD traffic flow along these two kinds of shortest-path. In section 4 and section 5 , we will compare the difference in assignment results according to these kinds of shortest-path.

The algorithm of these two kinds of shortest-path are almost the same as that of centroid-to-centroid shortest-path. The procedure pertains to the calculation of such as a minimum-path tree, that is the shortest-path from all nodes to one zone. The sortest path from all nodes in network to zone $J$ and the shortest-path from all zones to zone $J$ can be calculated as follws :

Step 1: Initialization. Let the travel cost from the node in zone $J$ to zone $J$ be zero, that is :

$$
S T_{k j}=0 \quad \forall k \in S N_{j}
$$

It is to say, when searching for the node-to-zone shortest-path from all nodes to zone $J$, the initialization step of the sortest-path algorithm should set the travel cost from all nodes in zone $J$ to zone $J$ to be zero. This is equal to the case that all of the nodes in zone $J$ are aggregated into a big node and then calculate the minimum-path tree from all other nodes in 
network to this big node in the following steps. It is known that the initialization step of the centroid-to-centroid shortest-path algorithm set the travel cost from the centroid of zone $J$ to the centroid of zone $J$ to be zero. This is the only difference between two kinds of shortest-path algorithm.

Step 2 : Iteration. The iteration step is completely the same as the general shortest-path algorithm. This can be found in Sheffi (1985). After the iteration step finished, all of the shortest-paths from all nodes in network to zone $J$ are found.

Step 3 : After the shortest-paths from all nodes in network to zone $J$ are found, the shortest-path from zone $I$ to zone $J$ can be found by comparing the node-to-zone shortest travel cost from each node in zone $I$ to zone $J$. That is :

$$
L_{i j}=\min _{k \in S N_{i}} S T_{k j}
$$

Compared to centroid-to-centroid shrotest-path algorithm, this step is the only additional calculation in zone-to-zone shortest-path algorithm. However, the calculational effort in this step is very few and can almost be omitted because the needed calculation in this step is only $m(m$ is the number of nodes in zone $I$ ) comparison.

From those procedures of shortest-path-searching, it can be concluded that the needed calculation to search for the shortest-paths from all nodes to all zones and to search for the shortest-path from all zones to all other zones are almost the same as that to search for the shortest-path from all centroids to all centroids.

\section{Improved Traffic Assignment Methods}

All of traffic assignment methods (for example, incremental assignment method or equilibrium assignment method) are a kind of iterative method. In each iteration, all or part of OD traffic flow is assigned to the shortest-path from origin to destination. Here, we have defined two new kinds of shortest-path, and correspondingly, we can develop two new kinds of traffic assignment methods.

\section{(1) To assign OD traffic flow along the zone-to-zone shortest-path}

In this paper, we call this method as zone-to-zone assignment method.

This method is almost the same as the general traffic assignment method, the only difference is that the OD traffic flow is assigned to the zone-to-zone shortest-path from origin zone to destination zone in each iteration of this method. That is to say, to replace the centroid-to-centroid shortest-path from origin centroid to destination centroid by the zone-tozone shortest-path from origin zone to destination zone, both incremental assignment method and equilibrium assignment method can be carried out too. The equilibrium assignment method by zone-to-zone shortest-path can be stated as follows : 


\section{AN IMPROVEMENT ON TRAFFIC ASSIGNMENT METHOD}

Step 0 : Initialization. Find the zone-to-zone shortest-path based on $t_{a}=t_{a}(0), \forall a$. Perform all-or-nothing assignment according to the zone-to-zone shortest-path. This yields $\left\{x_{a}^{1}\right\}$. Set counter $n=1$.

Step 1 : Update. Set $t_{a}^{n}=t_{a}\left(x_{a}^{n}\right), \forall a$.

Step 2 : Direction finding. Find the zone-to-zone shortest-path based on $t_{a}^{n}$. Perform all-or-nothing assignment according to the zone-to-zone shortest-path. This yields a set of (auxiliary) flows $\left\{y_{a}^{n}\right\}$.

Step 3 : Line search. Find $\alpha_{n}$ that solves

$$
\operatorname{Min}_{0 \leqq \alpha \leq 1} \sum_{a} \int_{0}^{x_{a}^{n}+\alpha\left(y_{a} a^{n} x_{a}^{n}\right)} t_{a}(\omega) d \omega
$$

Step 4 : Move. Set $x_{a}^{n+1}=x_{a}^{n}+\alpha_{n}\left(y_{a}^{n}-x_{a}^{n}\right), \forall a$.

Step 5 : Convergence test. If a convergence criterion is met, stop ; otherwise, set $n:=$ $n+1$ and go to step 1 .

In the above steps, $x_{a}$ is the traffic flow on link $a, y_{a}$ is the auxiliary link traffic flow of link $a$ and $t_{a}$ is the travel cost of link $a$.

Though it seems that this method is almost the same as general traffic assignment method, because the OD traffic flow is assigned to the network based on zone-to-zone shortest-path, this method can improve the accuracy of estimated link traffic flow greatly. The reason can be explained as follows.

First, the traffic of any zone can be generated from many nodes in the zone instead of from only one node. Since the traditional traffic assignment always assign OD traffic flow to the centroid-to-centroid shortest-path, all of the OD traffic of any zone is forced to be generated from the centroid of the zone. However, this improved assignment method assigns OD traffic flow to zone-to-zone shortest-path in each iteration, and the zone-to-zone shortestpath is actually the shortest-path from the border of one zone to the border of another zone. Therefore, the OD traffic of a zone may be generated from different nodes on the border of the zone. As a result, the overconcentration of traffic flow on the links near centroid in the traditional assignment method can be avoided in this improved assignment method. Second, the routes to which OD traffic flow may be loaded in this improved method are more close to the routes which may be used by the drivers in the actual network. Since the centroid-tocentroid shortest-path is calculated according to the travel cost from one centroid to another centroid, the travel cost of the routes near to centroids are less than that of the routes far to centroids. As a result, the routes near to centroids may be used often, but the routes far to centroids may be used rarely in the traditional assignment procedure. However, in this improved method, the zone-to-zone shortest-path is calculated according to the travel cost from the border of one zone to the border of another zone, each route between two zones is treated equally, and each route can be assigned reasonable traffic flow in the assignment 
procedure. For example, if the traditional assignment method is used to run the assignment problem of figure 3 , the feasible routes between two centroids, which are assumed to be node 4 and node 5 , may be the routes $4-2-6-5,4-3-7-5$ and 4-5. However, since the travel cost of route 4-2-6-5 and the travel cost of route 4-3-7-5 are much greater than that of route 4-5, it is almost impossible that the shortest path from node 4 to node 5 can include link 2-6 or link 3-7. As a result, all of the OD traffic may be assigned to link 4-5. In this improved method, the traffic assignment is based on zone-to-zone shortest-path, the feasible routes between two zones should be route $2-6$, route $4-5$ and route $3-7$. Since the travel cost of these three routes are close to each other, all of the link 4-5, link 2-6, and link 3-7 may be loaded to a certain value of traffic flow in this method. In the actual traffic network, the drivers between two zones may also travel along link 2-6 and link 3-7 because the OD traffic may be generated anywhere in the zone. Therefore, to assign OD traffic along the shortest-path from origin zone to destination zone is close to the real performance of traffic network.

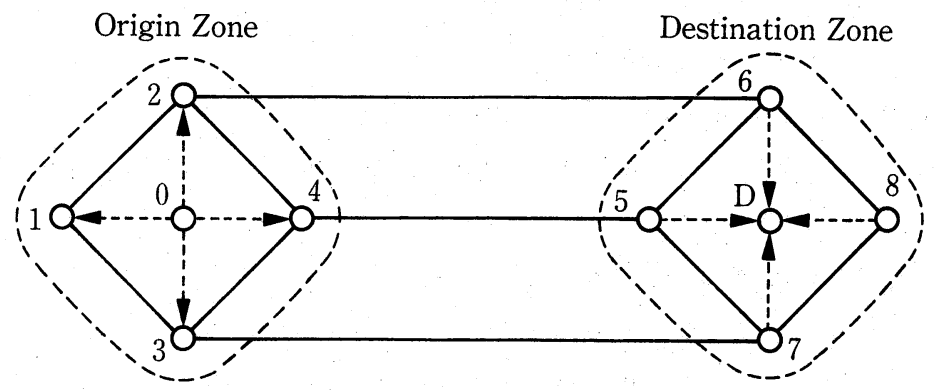

Figure 1. The equivalent network of centroid-to-centroid assignment method

This method can also get unique optimum assignment. Actually, this method is the same as the case that origin centroid is connected to all nodes in origin zone by a set of dummy links and the destination centroid is connected to all nodes in destination zone by a set of dummy links as in figure 1 . Since carrying out the traffic assignment according to figure 1 can surely obtain equilibrium assignment, this method can also obtain a unique equilibrium assignment.

\section{(2) To assign OD traffic flow along the node-to-zone shortest-path}

We call this method as node-to-zone assignment method.

In the real traffic network, traffic is actually generated from each node and destined to each node in the network. The most accurate assignment model should set each node to be origin node and destination node. However, either data collection or computer capacity do not allow to do so. Therefore, current assignment methods always aggregate some nodes into a zone and set one centroid in each zone. To do so can save the cost of data collection and calculation in expense of inaccuracy. Here, in order to improve the accuracy, we consider the following case : 


\section{AN IMPROVEMENT ON TRAFFIC ASSIGNMENT METHOD}

The network is still divided into many zones and OD traffic flow is still the traffic from origin zone to destination zone. However, when the OD traffic flow of a OD pair is assigned to the network, each node in origin zone is treated as an independent origin node, and all of the nodes in destination zone are aggregated into a big node and treated as a destination.

In the above case, the number of origins is equal to the number of nodes and the number of destinations is equal to the number of zones. We will explain that neither the needed data nor the needed calculation for this case will increase.

For the needed data of this case, we can use the same OD traffic flow from one zone to another zone in general traffic assignment method, and then distribute the total traffic of a zone to all the nodes in the zone according to certain proportion.

For the needed calculation of this case, it is known that finding the minimum paths is the most computation-intensive procedure in traffic assignment problem. However, the current shortest-path algorithm can find the minimum-path trees from all nodes in network to one destination simultaneously, and the number of minimum-path trees needed to be found in assignment procedure is equal to the number of destinations. If the number of destinations is the same, the computational effort for finding the minimum-path trees is also the same in spite of the difference in the number of origins. In the above case, though the number of origins has increase to be equal to the number of nodes, the number of destinations is still equal to the number of zones. Therefore, the needed calculation of this case will not increase.

The node-to-zone assignment method is corresponding to the above case, that is, the method assigns OD traffic flow to the node-to-zone shortest-path in each iteration.

The proportion of traffic flow distributed to the node in a zone can be assumed to be proportional to the capacity of all links connected to the node. If we assume that $C_{k}$ is the total capacity of all links connected to node $K, q_{i j}$ is the OD traffic flow from zone $I$ to zone $J$, then the traffic flow $\left(q^{\prime}{ }_{k j}\right)$ from node $K$ to zone $J$ can be calculated as :

$$
q^{\prime}{ }_{k j}=q_{i j} \times \frac{C_{k}}{\sum_{1 \in L N_{i}} C_{l}}
$$

There are two steps for this method. The first step is to distribute the OD traffic among the nodes in origin zone. The second step is to assign the traffic flow obtained in step 1 to the node-to-zone shortest-path from each node in origin zone to the destination zone. The second step is the same as the case that each node in origin zone is regarded as a origin node from which OD traffic flow can be generated independently. The amount of OD traffic flow to be generated from each origin node is the traffic flow distributed to the node in step 1 . The method is equivalent to the traffic assignment problem in figure 2. In the figure, each node in origin zone is an independent origin node, and there is only one centroid in destination zone which is connected to each node in the zone by a set of dummy links.

Since this method can be expressed as the equivalent assignment problem of the network 


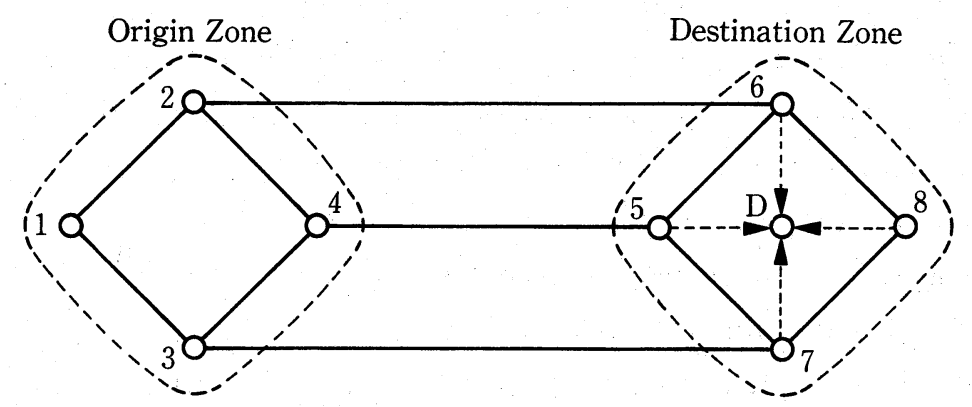

Figure 2. The equivalent network of centroid-to-centroid assignment method

in figure 2, this method can also obtain unique equilibrium assignment. As analyzed above, the needed data and calculation of node-to-zone assignment method are almost the same as that of general traffic assignment method. According to the above analysis, we can obtain the following conclusions about this method :

- The computational effort and the needed data of this method is the same as that of general traffic assignment methos.

- Since each node can generate a proportion of OD traffic flow, the results obtained by this method can be expected to be much more close to the performance of actual traffic network.

Here we presented two new kinds of shortest-path in traffic network. The OD traffic flow can be assigned to network according to the new shortest-path in each iteration of traffic assignment procedure. Two new kinds of traffic assignment method can be developed according to two kinds of shortest-path. Since the number of minimum-path trees needed to be found in each assignment iteration is equal to the number of destinations, and the number of destinations is equal to the number of zones for the two methods and the traditional assignment method, therefore, the computational effort needed by three methods is the same, and the necessary data for the three methods are also the same.

For the traditional assignment method, the traffic flow is always generated from the same node and destined to the same node in each assignment iteration for each OD pair, the way of trip generating and destining in the method is quite different from that in the actual traffic network.

For the second method, in one assignment iteration, the assigned traffic flow of one OD pair is generated from one node and destined to one node. However, in different assignment iteration, the OD traffic flow may be generated from different node and destined to different node. Therefore, for the final results of this method, the traffic flow in a zone may be generated from many or all nodes in the zone and may be destined to many or all nodes in the zone. To some extent, the second method agrees the performance of trip generating and 
destining in the actual traffic network. However, in this method, the proportion of OD traffic flow generated from each node is actually determined by the travel cost from each node to destination zone. If a node in orgin zone is near to a destination zone, most of the OD traffic flow of this OD pair may be generated from the node, the proportion of OD traffic generated from each node may be unreasonable in some cases.

In the third method, ti is assumed that the proportion of OD traffic flow generated from each node is determined by the total capacity of the links connected to the node, and the OD traffic flow is generated from each node in origin zone according to the determined ratio and can be destined to any node in destination zone, therefore, trip generating and destining in this method is nearest to that in the actual traffic network. We will check the fitness of these three methods in the following section.

\section{Evaluation of the Improved Traffic Assignment Methods}

In this paper, two improved traffic assignment methods were developed. It is expected that the zone-to-zone assignment methods and the node-to-zone assignment method are closer to the actual performance of traffic network than the centroid-to-centroid assignment method is. In order to check the efficiency of the three methods and to analyze the difference existed in three methods, a simple network as in figure 3 is used to run the assignment problem by three methods respectively.

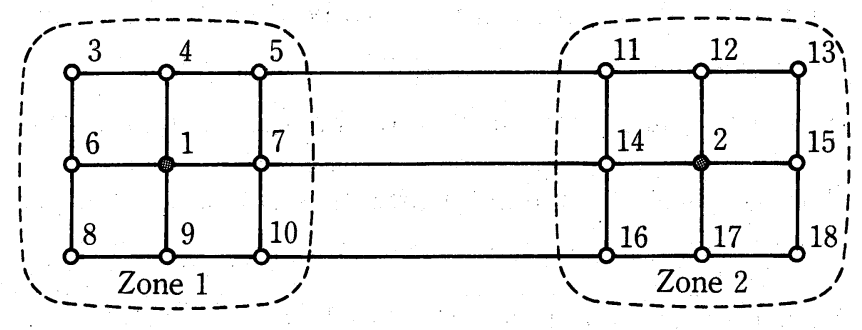

Figure 3. The assumed network

There are two zones in the network, that is zone 1 and zone 2 . There exist 9 nodes in zone 1 , that is node $1,3,4,5,6,7,8,9$ and 10 , and there also exist 9 nodes in zone 2 , that is node $2,11,12,13,14,15,16,17$ and 18. All of the links in the network are symmetric two-way links. The detail OD traffic flow from each node in zone 1 to each node in zone 2 is assumed to be 1. Therefore, the OD traffic flow from zone 1 to zone 2 is $1 \times 9 \times 9=81$. The free flow time and capacity of each link are assumed to be as in table 1. We take BPR function $t=t_{0}\{1+2.62$ $\left.\left(\frac{q}{C}\right)^{5}\right\}$ as link performance function. 
Kawakami and Liu

Table 1. The free flow time and the capacity of each link

\begin{tabular}{|c|r|r|r|r|r|r|r|r|r|r|r|r|r|r|}
\hline Link No. & $5-11$ & $7-14$ & $10-16$ & $3-4$ & $4-5$ & $6-1$ & $1-7$ & $8-9$ & $9-10$ & $6-3$ & $1-4$ & $7-5$ & $6-8$ & $1-9$ \\
\hline Free Flow Time & 10 & 10 & 10 & 3 & 3 & 3 & 3 & 3 & 3 & 3 & 3 & 3 & 3 & 3 \\
\hline Capacity & 40 & 40 & 40 & 30 & 30 & 30 & 30 & 30 & 30 & 30 & 30 & 30 & 30 & 30 \\
\hline Link No. & $7-10$ & $11-12$ & $12-13$ & $14-2$ & $2-15$ & $16-17$ & $17-18$ & $11-14$ & $12-2$ & $13-15$ & $16-14$ & $17-2$ & $18-15$ & \\
\hline Free Flow Time & 3 & 3 & 3 & 3 & 3 & 3 & 3 & 3 & 3 & 3 & 3 & 3 & 3 & \\
\hline Capacity & 30 & 30 & 30 & 30 & 30 & 30 & 30 & 30 & 30 & 30 & 30 & 30 & 30 & \\
\hline
\end{tabular}

We analyze what influence will cast on the assignment results if the traffic flow generated from each node is aggregated into the OD traffic flow from one zone. First, we use the detail OD traffic flow from each node in zone 1 to each node in zone 2 to run the assignment problem. In this case, each node in zone 1 is an origin and each node in zone 2 is a destination. Therefore, the number of OD pairs is $9 \times 9=81$, and the OD traffic flow for each OD pair is 1. The assignment results in this case can be regarded as the correct assignment, and the calculated link traffic flow is regarded to be correct value. Second, we aggregate the 9 nodes in zone 1 into one origin zone and aggregate the 9 nodes in zone 2 into one destination zone to run the assignment problem by three methods. The assignment methods will be evaluated by comparing the difference between the correct link traffic flow and the link traffic flow calculated by three assignment methods. For centroid-to-centroid assignment, origin centroid is set to be node 1 and destination centroid is set to be node 2 . The calculated traffic flow of each link by three methods is as in the table 2 .

First, we compare the calculated traffic flow of the inter-zone link, that is link 5-11, 7-14

Table 2. The comparison of the link traffic flow calculated by three methods

\begin{tabular}{|l|r|r|r|r|r|r|r|r|r|r|r|r|r|r|}
\hline \multicolumn{1}{|c|}{ Link No. } & $5-11$ & $7-14$ & $10-16$ & $3-4$ & $4-5$ & $6-1$ & $1-7$ & $8-9$ & $9-10$ & $6-3$ & $1-4$ & $7-5$ & $6-8$ & $1-9$ \\
\hline Correct Value & 27.0 & 27.0 & 27.0 & 9.0 & 18.0 & 9.0 & 18.0 & 9.0 & 18.0 & 2.87 & 3.28 & 2.94 & 3.0 & 3.0 \\
\hline $\begin{array}{l}\text { Centro id - to- } \\
\text { Centroid }\end{array}$ & 25.4 & 30.1 & 25.4 & 0.0 & 25.4 & 0.0 & 30.14 & 0.0 & 25.4 & 0.0 & 25.4 & 0.0 & 0.0 & 25.4 \\
\hline Zone-to-Zone & 27.0 & 27.0 & 27.0 & 0.0 & 0.0 & 0.0 & 0.0 & 0.0 & 0.0 & 0.0 & 0.0 & 0.0 & 0.0 & 0.0 \\
\hline Node-to Zone & 26.4 & 28.2 & 26.4 & 8.4 & 17.3 & 9.0 & 18.5 & 8.4 & 17.3 & 0.0 & 0.0 & 0.0 & 0.0 & 0.0 \\
\hline \hline \multicolumn{1}{|c|}{ Link No. } & $7-10$ & $11-12$ & $12-13$ & $14-2$ & $2-15$ & $16-17$ & $17-18$ & $11-14$ & $12-2$ & $13-15$ & $16-14$ & $17-2$ & $18-15$ & \\
\hline Correct Value & 2.9 & 18.0 & 9.0 & 18.0 & 9.0 & 18.0 & 9.0 & 3.0 & 3.0 & 2.9 & 3.1 & 3.0 & 3.0 & \\
\hline $\begin{array}{l}\text { Centroid-t o- } \\
\text { Centroid }\end{array}$ & 0.0 & 25.4 & 0.0 & 30.1 & 0.0 & 25.4 & 0.0 & 0.0 & 25.4 & 0.0 & 0.0 & 25.4 & 0.0 & \\
\hline Zone-to-Zone & 0.0 & 0.0 & 0.0 & 0.0 & 0.0 & 0.0 & 0.0 & 0.0 & 0.0 & 0.0 & 0.0 & 0.0 & 0.0 & \\
\hline Node-to-Zone & 0.0 & 0.0 & 0.0 & 0.0 & 0.0 & 0.0 & 0.0 & 0.0 & 0.0 & 0.0 & 0.0 & 0.0 & 0.0 & \\
\hline
\end{tabular}


and 10-16. From table 2, it can be seen that the calculated link traffic flow of the three links by both zone-to-zone assignment method and node-to-zone assignment method is very close to the correct value. The percent error for three inter-zone links by zone-to-zone assignment method is $0 . \%, 0 . \%$ and $0 . \%$, and the percent error for three inter-zone links by node-to-zone assignment method is $4.4 \%,-2.2 \%$ and $-2.2 \%$ respectively. For the centroid-to-centroid assignment method, the percent error for three inter-zone links is $11.5 \%,-5.9 \%,-5.9 \%$, which is much greater than that produced by two improved methods. We also calculated the case that the centroids are set to be other nodes by centroid-to-centroid assignment method. The maximum errors are greater than $11.5 \%$, and the calculated results are quite different from each other when the centroids are set to be different nodes. This difference also reflects that the assignment results by centroid-to-centroid assignment method are sensitive to the location of the centroid. There is no problem about the location of centroid in the other two methods because no centroid is needed in the improved methods.

Second, we compare the calculated traffic flow of the intra-zone link, that is all of the links except inter-zone link. Neither of these methods can accurately estimate the traffic flow of intra-zone links. The error is great for any of these methods. However, among these methods, the node-to-zone assignment method produced best estimation.

It is known that the analysis of the flows in the urban area does not focus on the flows within each traffic zone and the assignment method has to ensure only that the flows between the traffic zone are corect. The zone-to-zone assignment method and node-to-zone assignment method can produce better estimation on the traffic flows of inter-zone links, these two methods can be regarded as the good assignment methods.

As for computational time needed by each method, as expected above, the CPC time is almost the same for the three methods. In the case of one OD pair as in figure 3 , the CPU time on FACOM-780 is $0.34,0.31,0.41$ seconds for centroid-to-centroid, zone-to-zone, and node-to-zone assignment method respectively. However, to produce the correct value by running the 81 OD pairs assignment problem needs the CPU time of 16.52 seconds.

We can also analyze the difference in the final equilibrium assignment of the three methods from the calculated results. For the centroid-to-centroid assignment method, the final assignment results are the equilibrium among the routes from centroid to centroid, and the used routes have the same travel cost from origin centroid to destination centroid. In this example, the travel costs of route 1-7-14-2, route 1-4-5-11-12-2, and route 1-9-10-16-17-2 are the same. For the zone-to-zone assignment method, the final assignment results are the equilibrium among the routes from zone to zone, and the used routes have the same travel cost from origin zone to destination zone. In this example, the travel costs of route 5-11, route 7-14, and route 10-16 are the same. For the node-to-zone assignment method, the final assignment results are the equilibrium among the routes from the node in origin zone to 


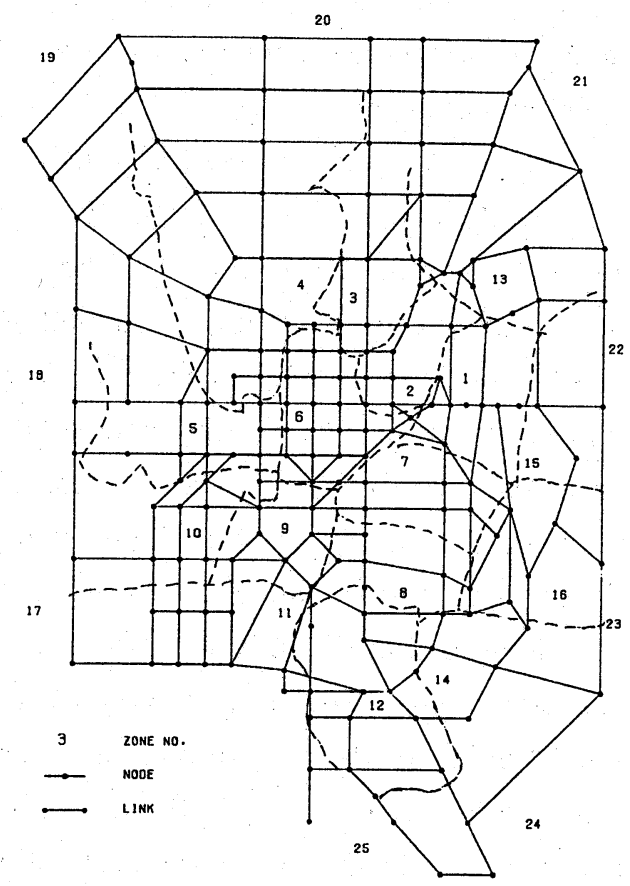

Figure 4. The road network of Nagoya city

destination zone. And the used routes have the same travel cost from the same node in origin zone to the same destination zone.

The traffic network of Nagoya city is also used to run the traffic assignment problem by three methods. The network is as in figure 4. The network, which includes 181 nodes, 634 links and 25 zones, is nearly equivalent to the arterial road network of Nagoya city. The OD traffic flow is obtained from person trip survey in 1986. The observed link traffic flow is obtained from road traffic census in 1985. The agreement between estimated link flows and the observed flows for three kinds of assignment method is shown in figure 5 (a), (b), and (c) separately.

From the figure 5 , it can be seen that the fitness of node-to-zone assignment method is best, almost all of the points in figure 5 (c) distribute around $45^{\circ}$ line; The fitness of zone-to-zone assignment method is also good, most of the points in figure 5 (b) distribute around $45^{\circ}$ line, but a few of points distribute in the area under $45^{\circ}$ line. The reason of this underestimation may be that the traffic flow generated from some nodes in this assignment method is not equal to that in the actual network ; The fitness of traditional centroid-tocentroid assignment method is not as good as two improved methods. Though most of points in figure 5 (a) still distribute along $45^{\circ}$ line, many points are far below or far above $45^{\circ}$ line. The reason of this estimation errors may be that the traffic flow assigned to the link near 


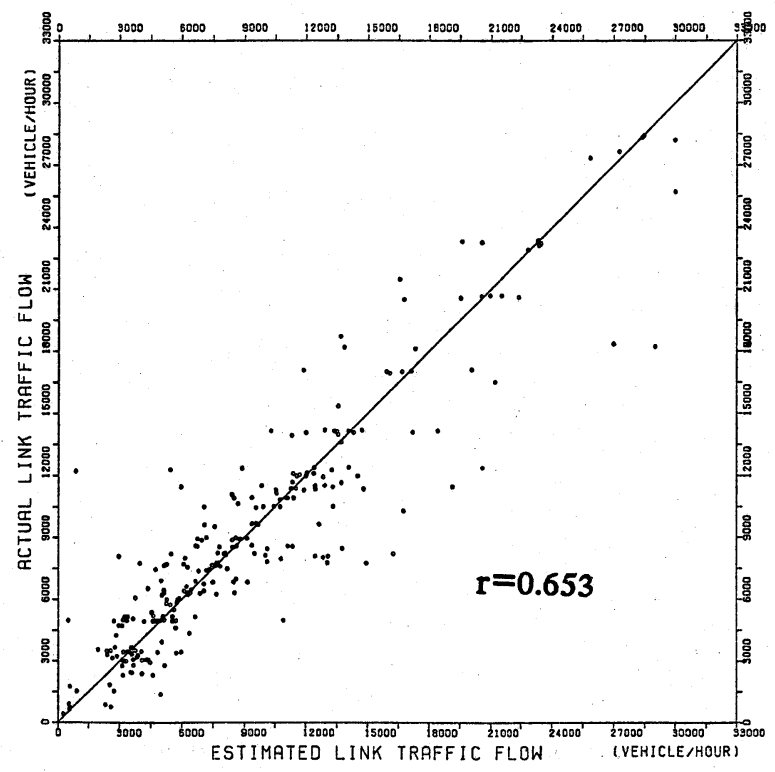

Figure 5(a). Node-to-node assignment

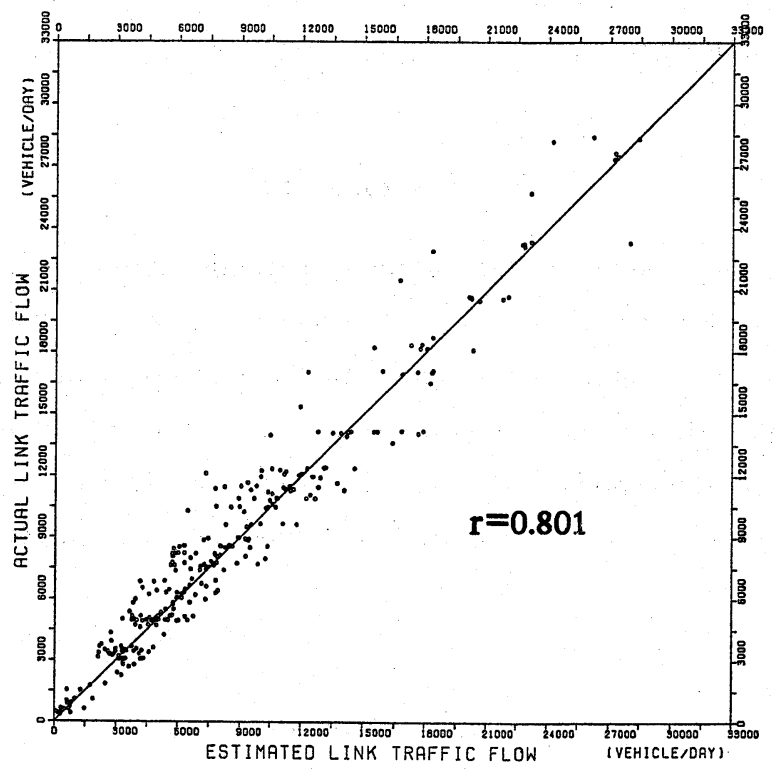

Figure 5(b). Zone-to-zone assignment 
Kawakami and Liu

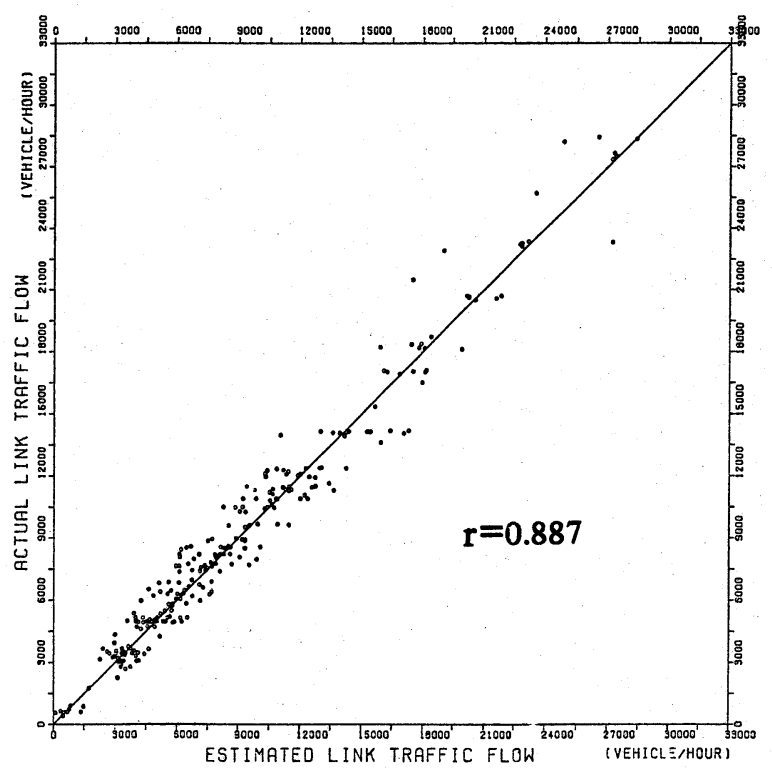

Figure 5(c). Node-to-zone assignment

Figure 5. The agreement of estimated link traffic flow by three kinds of assignment method

to centroid in this method is much more than the actual traffic flow of the link and the traffic flow assigned to the link far from centroid is less than the actual traffic flow of the link.

\section{Conclusions}

In this paper, two improved traffic assignment methods were developed. Since all nodes can generate and absorb traffic flow in the two methods, the traffic generation characteristic in traffic network can be reflected more really than the current traffic assignment methods do. Through the comparison of the calculated results of two networks by the methods, it can be seen that the improved methods are better than the current traffic assignment method, but the needed data and computation are almost the same as that of current traffic assignment method.

\section{References}

Daganzo C. F., 1980, Network representation, continuum approximation and a solution to the spatial aggregation problem of traffic assignment, Transpn. Res., vol. 14B, 229-239.

Iida, Y., Y. Asakura, H. Yang and H. Shinji, 1989, Traffic assignment by continuum approximation of network (In Japanese), Proceeding of Infrastructure Planning, no. 12, 543-550. 


\section{AN IMPROVEMENT ON TRAFFIC ASSIGNMENT METHOD}

Iida, Y., T. Akiyama and T. Uchid, 1989, Experimental analysis of dynamic route choice behavior analysis, International Conference on Dynamic Travel Bahavior Analysis (Preprints ; held in Kyoto, Japan), 5-4.

Kawakami S., Y. Hirobata and Z. Xu, 1989, An study on the multi-class traffic assignment method with separation of car and truck link flows(In Japanese), Infrastructure Planning Review, no. 7, 243-250.

Leblanc, 1975, An efficient approach to solving the road network equilibrium traffic assignment problem, Transpn. Res., vol. 9, 309-318.

Matsui H. and T. Niwa, 1986, A mathematical analysis of route guidance in the network(In Japanese), Infrastructure Planning Review, no. 4, 85-92.

Sheffi Y., 1985, Urban Transportation Networks : Equilibrium Analysis with Mathematical Programming Methods, Prentice-Hall, New Jersey.

Shimazaki T. and Y. Matsumoto, 1989, Simulation of personal route choice mechanism, International Conference on Dynamic Travel Behavior Analysis (Preprints ; held in Kyoto, Japan), 5-3. 